\title{
Rotating Variable-Thickness Inhomogeneous Cylinders: Part II-Viscoelastic Solutions and Applications
}

\author{
Ashraf M. Zenkour \\ Department of Mathematics, Faculty of Science, King AbdulAziz University, Jeddah, Saudi, Saudi Arabia \\ Department of Mathematics, Faculty of Science, Kafrelsheikh University, Kafr el-Sheikh, Egypt \\ E-mail: zenkour@gmail.com \\ Received June 2, 2010; revised October 12, 2010; accepted October 16, 2010
}

\begin{abstract}
Analytical solutions for the rotating variable-thickness inhomogeneous, orthotropic, hollow cylinders under plane strain assumption are developed in Part I of this paper. The extensions of these solutions to the viscoelastic case are discussed here. The method of effective moduli and Illyushin's approximation method are used for this purpose. The rotating fiber-reinforced viscoelastic homogeneous isotropic hollow cylinders with uniform thickness are obtained as special cases of the studied problem. Numerical application examples are given for the dimensionless displacement of and stresses in the different cylinders. The influences of time, constitutive parameter and elastic properties on the stresses and displacement are investigated.
\end{abstract}

Keywords: Rotating, Viscoelastic Cylinder, Orthotropic, Variable Thickness and Density

\section{Introduction}

In recent years the subject of viscoelasticity has received considerable attention from analysts and experimentalists. The stress state of a viscoelastic hollow cylinder with the help of internal pressure and temperature field is analyzed in the literature $[1,2]$. A modified numerical method is introduced by Ting and Tuan [3] to study the effect of cyclic internal pressure on the stress and temperature distributions in a viscoelastic cylinder. Talybly [4] has investigated the state of stress and strain for a viscoelastic hollow cylinder fastened to an elastic shell under nonisothermal dynamic loading. Feng et al. [5] have obtained the solution for finite deformations of a viscoelastic solid cylinder subjected to extension and torsion. The thermomechanical behavior of a viscoelastic finite circular cylinder under axial harmonic deformations is presented by Karnaukhov and Senchenkov [6].

The determination of stress and displacement fields is an important problem in design of engineering structures using fiber-reinforced composite materials. The analytical solution for the rotating fiber-reinforced viscoelastic cylinders becomes very complex when the thickness along the radius of the cylinder is variable, even for simple cases. Methods for solving quasi-static viscoelastic problems in composite structures have been developed by a number of authors [7-9]. Allam and Appleby [10] have used the realization method of elastic solutions to solve the problem of bending of a viscoelastic plate reinforced by unidirectionally elastic fibers. In other work [11], they have used the method of effective moduli to determine the stress concentrations around a circular hole or circular inclusion in a fiber-reinforced viscoelastic plate under uniform shear. Allam and Zenkour [12] have used the small parameter method as well as the method of effective moduli for the bending response of a fiberreinforced viscoelastic arched bridge model with quadratic thickness variation and subjected to uniform loading. In [13], they have also obtained the stresses around filled and unfilled circular holes in a fiber-reinforced viscoelastic plate under bending. The same author [14] have developed closed form solutions for the rotating fiberreinforced viscoelastic solid and annular disks with variable thickness by applying the generalization of Illyushin's approximation method. In addition, Allam et al. [15] have determined the stress concentrations around a triangular hole in a fiber-reinforced viscoelastic composite plate under uniform tension or pure bending. Also, Zenkour et al. [16] have presented the elastic and viscoelastic solutions to rotating functionally graded hollow and solid cylinders.

In the present paper, the rotating fiber-reinforced viscoelastic hollow cylinder is analytically studied. The thickness of the cylinder and the elastic properties are 
taken to be functions in the radial coordinate. The governing second-order differential equation is derived and solved with the aid of some hypergeometric functions. The displacement and stresses for rotating fiberreinforced viscoelastic inhomogeneous orthotropic hollow cylinder with variable thickness and density subjected to various boundary conditions are obtained. Special cases of the studied problem are established and numerical results are presented in graphical forms.

\section{Rotation of Viscoelastic Cylinders}

According to the elastic solution given in part I, we can use the method of effective moduli and Illyushin's approximation method to solve the rotation problem of variable thickness and density viscoelastic hollow cylinder reinforced with unidirectionally elastic fibers.

For an orthotropic cylinder, the compliance parameters $\alpha_{i j}$ can be expressed in terms of the engineering characteristics as [17]:

$$
\begin{aligned}
& \alpha_{11}=\frac{E_{r}\left(1-v_{\theta z} v_{z \theta}\right)}{\Delta}, \alpha_{22}=\frac{E_{\theta}\left(1-v_{r z} v_{z r}\right)}{\Delta}, \\
& \alpha_{12}=\frac{E_{r}\left(v_{\theta r}+v_{\theta z} v_{z r}\right)}{\Delta}=\frac{E_{\theta}\left(v_{r \theta}+v_{z \theta} v_{r z}\right)}{\Delta}, \\
& \alpha_{13}=\frac{E_{r}\left(v_{z r}+v_{\theta r} v_{z \theta}\right)}{\Delta}=\frac{E_{z}\left(v_{r z}+v_{r \theta} v_{\theta z}\right)}{\Delta}, \\
& \alpha_{23}=\frac{E_{\theta}\left(v_{z \theta}+v_{r \theta} v_{z r}\right)}{\Delta}=\frac{E_{z}\left(v_{\theta z}+v_{\theta r} v_{r z}\right)}{\Delta},
\end{aligned}
$$

in which

$$
\Delta=1-v_{r \theta} v_{\theta r}-v_{z r} v_{r z}-v_{\theta z} v_{z \theta}-2 v_{\theta r} v_{z \theta} v_{r z},
$$

where $E_{i}$ are Young's moduli and $v_{i j}$ are Poisson's rations which are related by the reciprocal relations:

$$
\frac{v_{\theta r}}{E_{\theta}}=\frac{v_{r \theta}}{E_{r}}, \quad \frac{v_{r z}}{E_{r}}=\frac{v_{z r}}{E_{z}}, \quad \frac{v_{\theta z}}{E_{\theta}}=\frac{v_{z \theta}}{E_{z}} .
$$

Now, consider a hollow cylinder made of a composite material composed of two components. A viscoelastic material as a first component, reinforced by unidirectional elastic fibers as a second component. The first of these components plays the role of filler and may posses the properties of a linear viscoelastic material, and it is described by the modulus $E_{f}$ and Poisson's ratio $v_{f}$. The other component will be serve as the reinforcement and is an elastic material with modulus of elasticity $E$ and Poisson's ratio $v$.

Under the above considerations and using the method of effective moduli [14,18], Young's moduli and Poisson's ratios, with $v_{r \theta}=v_{\theta r}=v_{r z}=v_{\theta z}=v_{1}$ and $v_{z r}=v_{z \theta}=v_{2}$, are given by [19]:

$$
\begin{aligned}
& E_{r}=E_{\theta}=\frac{E E_{f}}{\gamma E_{f}+(1-\gamma) E}, \quad E_{z}=\gamma E+(1-\gamma) E_{f}, \\
& v_{1}=\frac{\left[\gamma \nu+(1-\gamma) v_{f}\right] E E_{f}}{\left[\gamma E+(1-\gamma) E_{f}\right]\left[\gamma E_{f}+(1-\gamma) E\right]}, \quad v_{2}=\gamma v+(1-\gamma) v_{f}
\end{aligned}
$$

where $\gamma$ is the volume fraction of fiber reinforcement. Thus, it is obvious that the reciprocal relations given in Equation (3) are fulfilled.

Note that, the viscoelastic modulus $E_{f}$ is given by:

$$
E_{f}=\frac{9 K \omega}{2+\omega},
$$

where $K$ is the coefficient of volume compression (the bulk modulus) and it is assumed to be not relaxed, i.e. $K=$ const., and $\omega$ is the dimensionless kernel of relaxation function which is related to the corresponding Poisson's ratio by the formula:

$$
\omega=\frac{1-2 v_{f}}{1+v_{f}} .
$$

Substituting from Equations (5) and (6) into Equation (4) yields

$$
\begin{aligned}
& E_{r}=E_{\theta}=E\left[\frac{9 p \omega}{2(1-\gamma)+(1-\gamma+9 p \gamma) \omega}\right], E_{z}=E\left[\gamma+\frac{9(1-\gamma) p \omega}{2+\omega}\right], \\
& v_{1}=\frac{\left[\gamma \nu+\frac{(1-\gamma)(1-\omega)}{2+\omega}\right] \frac{9 p \omega}{2+\omega}}{\left[\gamma+\frac{9(1-\gamma) p \omega}{2+\omega}\right]\left[\frac{9 p \gamma \omega}{2+\omega}+1-\gamma\right]}, v_{2}=\gamma \nu+\frac{(1-\gamma)(1-\omega)}{2+\omega}
\end{aligned}
$$

or in the simple form

$$
\begin{aligned}
& E_{r}=E_{\theta}=\frac{9 E p}{2(1-\gamma) \beta_{2}}\left(1-g_{\beta_{2}}\right), \quad E_{z}=E\left[\gamma+9 p(1-\gamma)\left(1-g_{\beta_{1}}\right)\right], \\
& v_{1}=\frac{9 p\left[\gamma v-(1-\gamma)\left(1-\frac{3}{2} g_{\beta_{1}}\right)\right]\left(1-g_{\beta_{1}}\right)}{\left[\gamma+9 p(1-\gamma)\left(1-g_{\beta_{1}}\right)\right]\left[9 p \gamma\left(1-g_{\beta_{1}}\right)+1-\gamma\right]}, \\
& v_{2}=\gamma v-(1-\gamma)\left(1-\frac{3}{2} g_{\beta_{1}}\right),
\end{aligned}
$$

in which

$$
g_{\beta_{i}}=\frac{1}{1+\beta_{i} \omega}, \beta_{1}=\frac{1}{2}, \beta_{2}=\frac{1}{2}\left(1+\frac{9 p \gamma}{1-\gamma}\right),
$$

where $p=K / E$ is the constitutive parameter.

With the help of Equations (1) and (8), one can rewrite the solutions given in Part I of this paper; see Equations (20) and (23)-(25); in the form:

$$
\begin{aligned}
& u^{*}=\bar{\Omega}^{2} u(\bar{r}, \omega), \quad \sigma_{r r}^{*}=\hat{\Omega}^{2} \sigma_{r r}(\bar{r}, \omega), \\
& \sigma_{\theta \theta}^{*}=\hat{\Omega}^{2} \sigma_{\theta \theta}(\bar{r}, \omega), \quad \sigma_{z z}^{*}=\hat{\Omega}^{2} \sigma_{z z}(\bar{r}, \omega),
\end{aligned}
$$

where 


$$
\bar{\Omega}^{2}=\frac{\rho_{0} \Omega^{2} b^{3}}{E}, \quad \hat{\Omega}^{2}=\rho_{0} \Omega^{2} b^{2} .
$$

It is to be noted that, in elastic composites, the radial displacement and stresses are functions of $\omega$ and $\bar{r}$ while in viscoelastic composites they are operator functions of the time $t$ and $\bar{r}$. According to Illyushin's approximation method $[11,19,20]$, the function $u$ can be represented in the form:

$$
u(\bar{r}, \omega)=\sum_{i=1}^{5} A_{i}(\bar{r}) \Phi_{i}(\omega),
$$

where $\Phi_{i}(\omega)$ are some known kernels, constructed on the base of the kernel $\omega$ and may be chosen in the form:

$$
\Phi_{1}=1, \quad \Phi_{2}=\omega, \quad \Phi_{3}=\pi=\frac{1}{\omega}, \quad \Phi_{4}=g_{\beta_{1}}, \quad \Phi_{5}=g_{\beta_{2}},
$$

where $g_{\beta_{i}},(i=1,2)$ are given in Equation (9).

The coefficients $A_{i}(\bar{r})$ are determined from the system of algebraic equations

$$
\sum_{j=1}^{5} L_{i j} A_{j}=B_{i}, \quad(i=1, \cdots, 5),
$$

where

$$
L_{i j}=\int_{0}^{1} \Phi_{i} \Phi_{j} d \omega, \quad B_{i}=\int_{0}^{1} \Phi_{i} u(\bar{r}, \omega) d \omega .
$$

Now, let us consider the relaxation function in an exponential form

$$
\omega(t)=c_{1}+c_{2} e^{-\alpha t},
$$

where $c_{1}$ and $c_{2}$ are constants to be experimentally determined. Laplace-Carson transform can be used to determine the functions $\pi(t)$ and $g_{\beta_{i}}(t)$. Denoting the transforms of $\pi(t)$ and $g_{\beta_{i}}(t)$ by $\pi^{*}(t)$ and $g_{\beta_{i}}^{*}(t)$, since the transform of $\omega(t)$ is

$$
\omega^{*}(s)=c_{1}+\frac{s c_{2}}{s+\alpha},
$$

thus, we get

$$
\begin{aligned}
& \pi(t)=\frac{1}{c_{1}}\left[1-\frac{c_{2}}{c_{1}+c_{2}} e^{-c_{1} \tau /\left(c_{1}+c_{2}\right)}\right], \quad(\tau=\alpha t), \\
& g_{\beta_{i}}(t)=\frac{1}{1+\beta_{i} c_{1}}\left[1-\frac{\beta_{i} c_{2}}{1+\beta_{i}\left(c_{1}+c_{2}\right)} e^{-\left(1+\beta_{i} c_{1}\right) \tau\left[1+\beta_{i}\left(c_{1}+c_{2}\right)\right]}\right]
\end{aligned}
$$

Equation (12) for a viscoelastic composite may be recorded to obtain explicit formula for the radial displacement as function of $\bar{r}$ and time $t$ in the form:

$$
\begin{aligned}
& u^{*}(\bar{r}, t)=A_{1}(\bar{r}) \bar{\Omega}^{2}(t)+A_{2}(\bar{r}) \int_{0}^{t} \omega(t-\tau) d \bar{\Omega}^{2}(\tau) \\
& +A_{3}(\bar{r}) \int_{0}^{t} \pi(t-\tau) d \bar{\Omega}^{2}(\tau)+A_{4}(\bar{r}) \int_{0}^{t} g_{\beta_{1}}(t-\tau) d \bar{\Omega}^{2} \bar{\Omega}^{2}(\tau) \\
& +A_{5}(\bar{r}) \int_{0}^{t} g_{\beta_{2}}(t-\tau) d \bar{\Omega}^{2}(\tau) .
\end{aligned}
$$

Taking $\bar{\Omega}^{2}(t)=\bar{\Omega}_{0}^{2} H(t)$, where $H(t)$ is the Heaviside's unit step function given by

$$
H(t)= \begin{cases}1 & \text { if } \quad t \geq 0 \\ 0 & \text { if } \quad t<0\end{cases}
$$

Then, Equation (19) takes the form

$$
\begin{aligned}
& u^{*}(\bar{r}, t)= \\
& \bar{\Omega}_{0}^{2}\left\{A_{1} H(t)+A_{2} \omega(t)+A_{3} \pi(t)+A_{4} g_{\beta_{1}}(t)+A_{5} g_{\beta_{2}}(t)\right\} .
\end{aligned}
$$

where $\omega(t), \pi(t)$ and $g_{\beta_{i}}(t)$ are given in Equations (16) and (18). Using the same technique once again to obtain the radial, circumferential and axial stresses for the rotating fiber-reinforced viscoelastic hollow cylinder with variable thickness and density by replacing only $u^{*}(\bar{r}, t)$ with $\sigma^{*}(\bar{r}, t)$ and making the suitable changes in this case.

\section{Applications}

In this section, some numerical examples for the rotating fiber-reinforced viscoelastic inhomogeneous variablethickness cylinder will be introduced. The results of the present problem will be given for three sets of geometric parameters $k$ and $n$ for the thickness profile. The numerical applications will be carried out for the radial displacement and stresses that being reported herein are in the following dimensionless forms:

$$
u_{r}=\frac{u^{*}}{\bar{\Omega}_{0}^{2}}, \sigma_{r}=\frac{\sigma_{r r}^{*}}{\hat{\Omega}_{0}^{2}}, \sigma_{\theta}=\frac{\sigma_{\theta \theta}^{*}}{\hat{\Omega}_{0}^{2}}, \sigma_{z}=\frac{\sigma_{z z}^{*}}{\hat{\Omega}_{0}^{2}} .
$$

The effect of the elastic properties of the cylinder, constitutive and time parameters on the dimensionless radial displacement and stresses will be shown. The calculations will be carried out for the following values of parameters: $v=0.3, \gamma=c_{1}=0.1, c_{2}=0.9$ and $\omega=0.5$. In addition, other parameters are taken (except otherwise stated) as: $p=0.2, k=2.5, n=0.8$ and $m=1$. Also, the coefficient $\alpha$ is still unknown and the time parameter $\tau(\equiv \alpha t)$ is given in terms of it.

The distributions of the dimensionless stresses and displacement through the radial direction of the rotating fiber-reinforced viscoelastic inhomogeneous variablethickness cylinder are plotted in Figures 1-3 according to the FF, CC, FC and CF boundary conditions, respectively. For all hollow cylinders, the dimensionless radial displacement $u_{r}$ is the largest in the same position for small $k$, i.e. $k=0.6$. For $\mathrm{FF}$ and $\mathrm{CF}$ hollow cylinders, the dimensionless stresses are the largest for small $n$. The minimum values of the dimensionless radial stress $\sigma_{r}$ at the outer surface of the CC and FC hollow cylinders are larger for $k=0.6$. Also, 

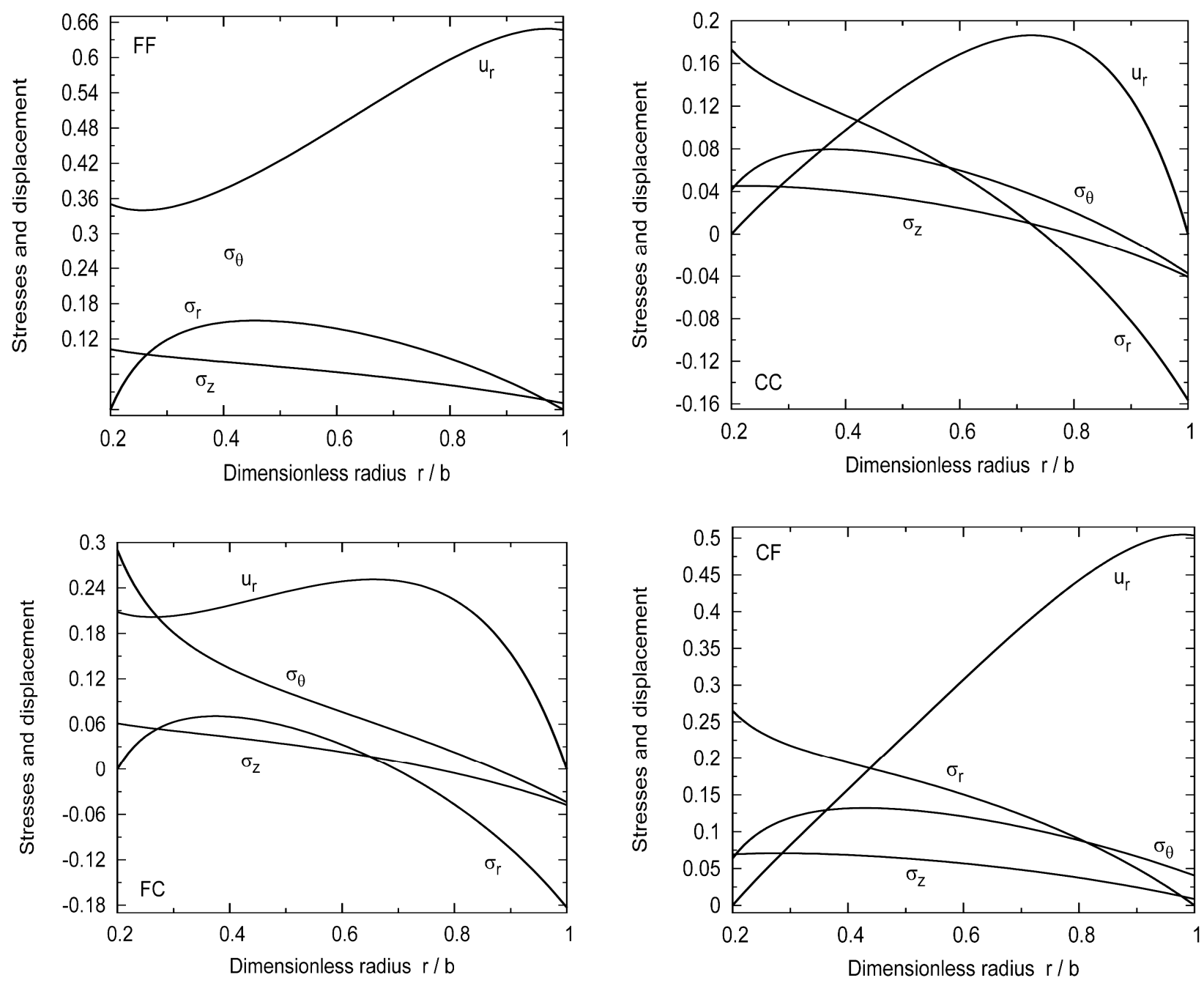

Figure 1. Dimensionless stresses and displacement for a variable-thickness viscoelastic hollow cylinder subjected to various boundary conditions $(k=0.6, n=0.8, m=0.5)$.
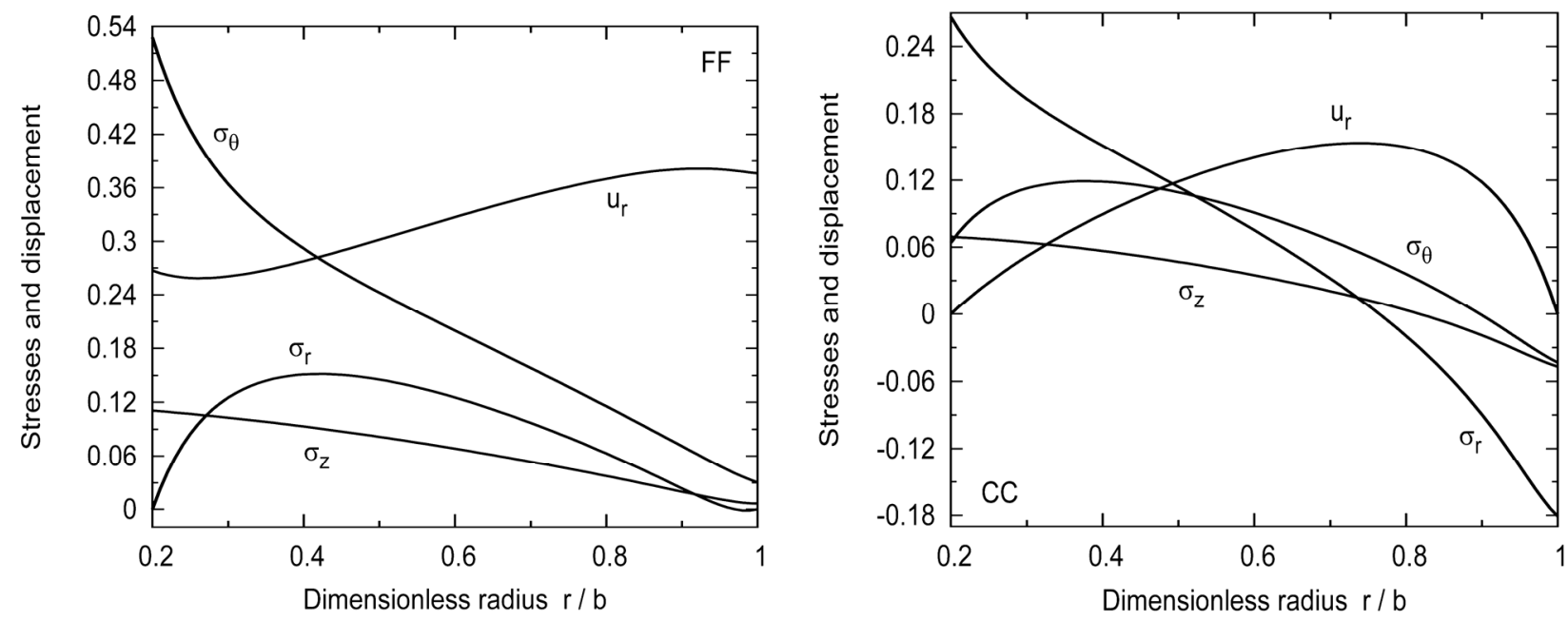

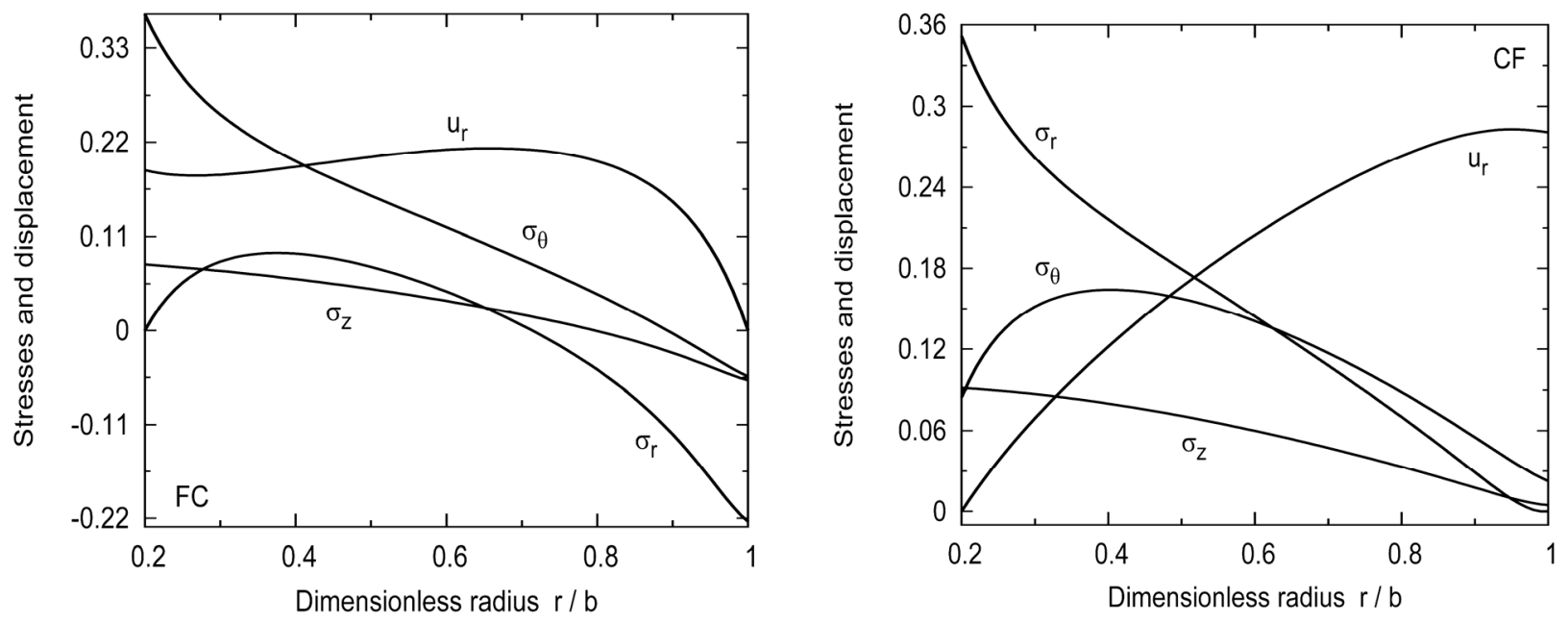

Figure 2. Dimensionless stresses and displacement for a variable-thickness viscoelastic hollow cylinder subjected to various boundary conditions $(k=2.5, n=0.8, m=0.5)$.
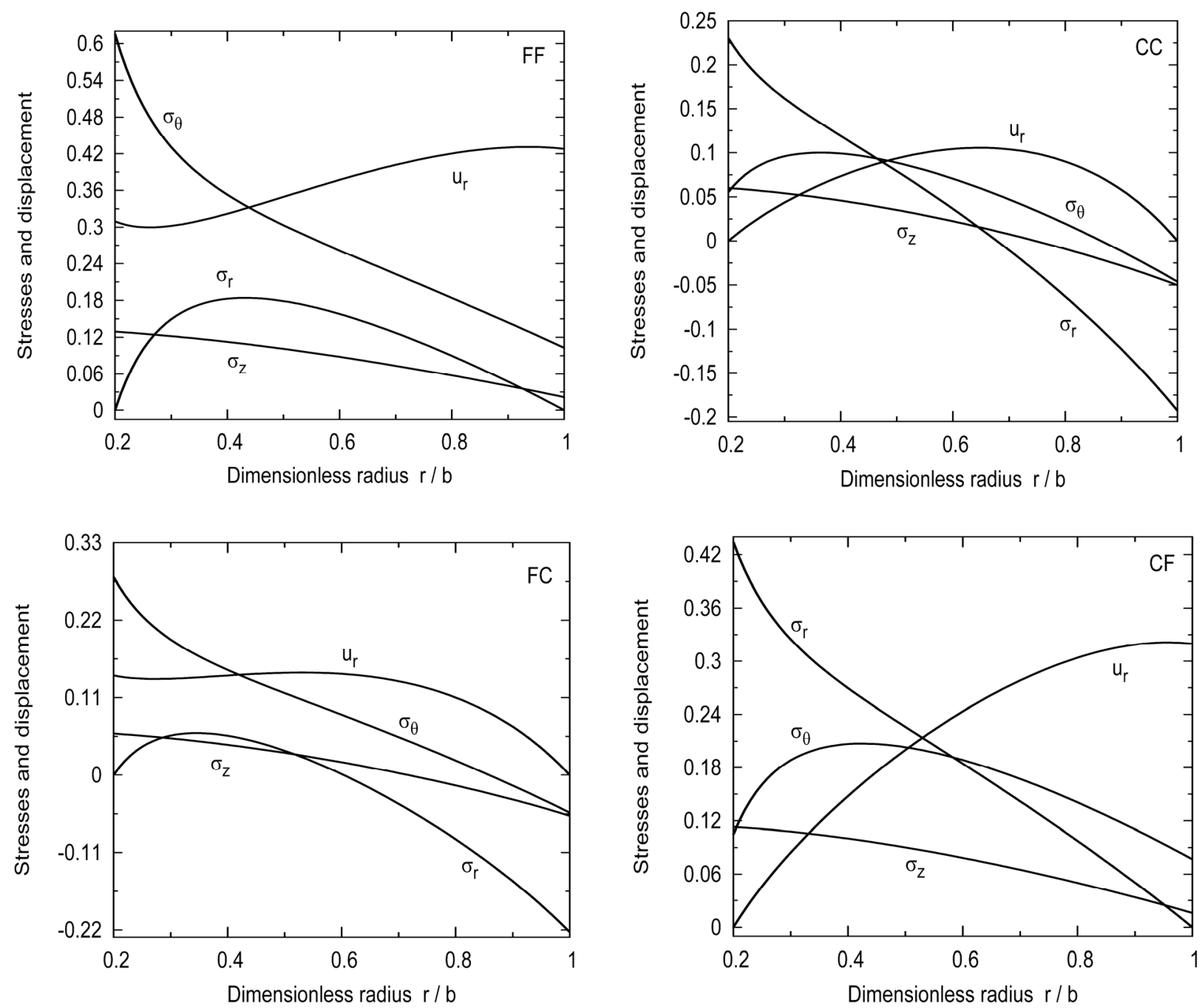

Figure 3. Dimensionless stresses and displacement for a variable-thickness viscoelastic hollow cylinder subjected to various boundary conditions $(k=2.5, n=0.4, m=0.5)$. 
the dimensionless circumferential $\sigma_{\theta}$ and axial $\sigma_{z}$ stresses are smaller through the radial direction of the CC hollow cylinders when $k=0.6$. The maximum value of $\sigma_{\theta}$ at the inner surface for FC hollow cylinder are the smallest when $k=2.5$ and $n=0.8$. In addition, the di-
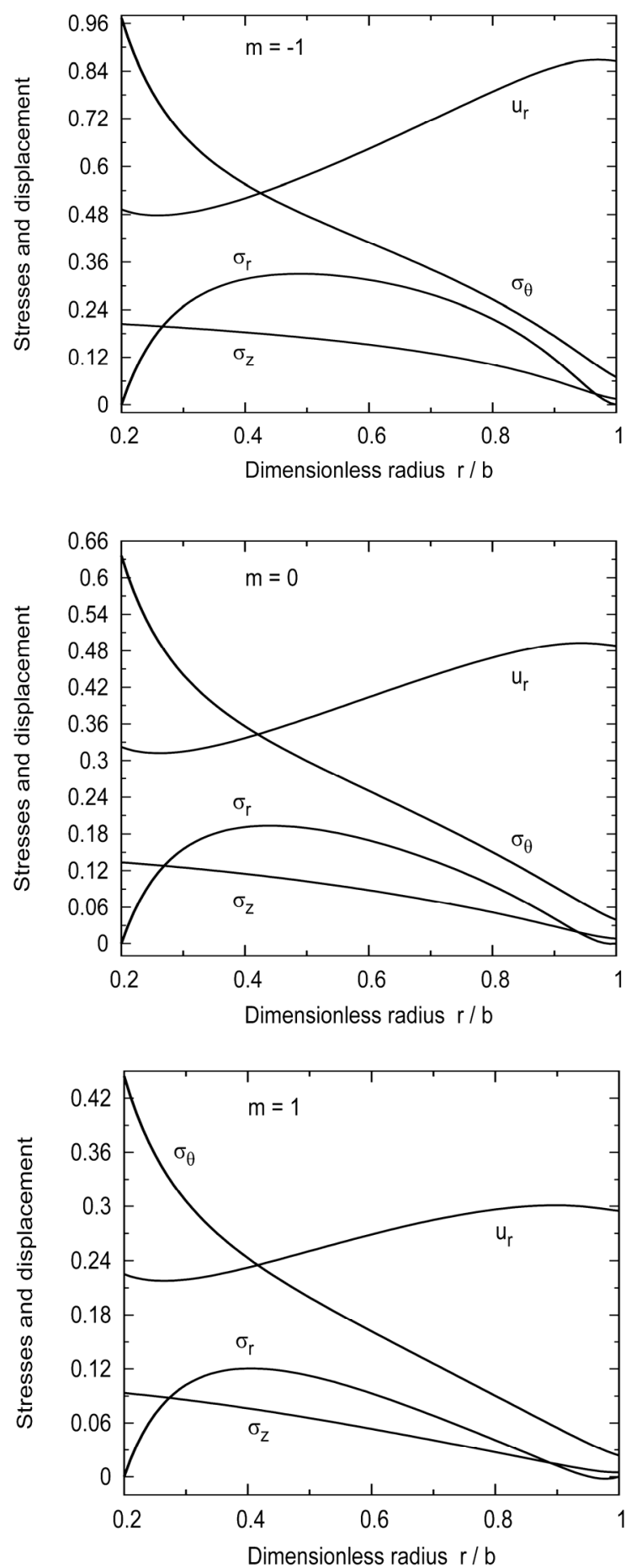

Figure 4. Distribution of dimensionless stresses and displacement through the radial direction of a FF variablethickness viscoelastic hollow cylinder. mensionless axial stresses are monotone decreasing in $\bar{r}$ and it is smaller for $n=0.4$ than for $n=0.8$.

For a profile with geometric parameters $k=2.5$ and $n=0.8$, the dimensionless displacement and stresses are plotted in Figures 4-7 for the rotating fiber-reinforced
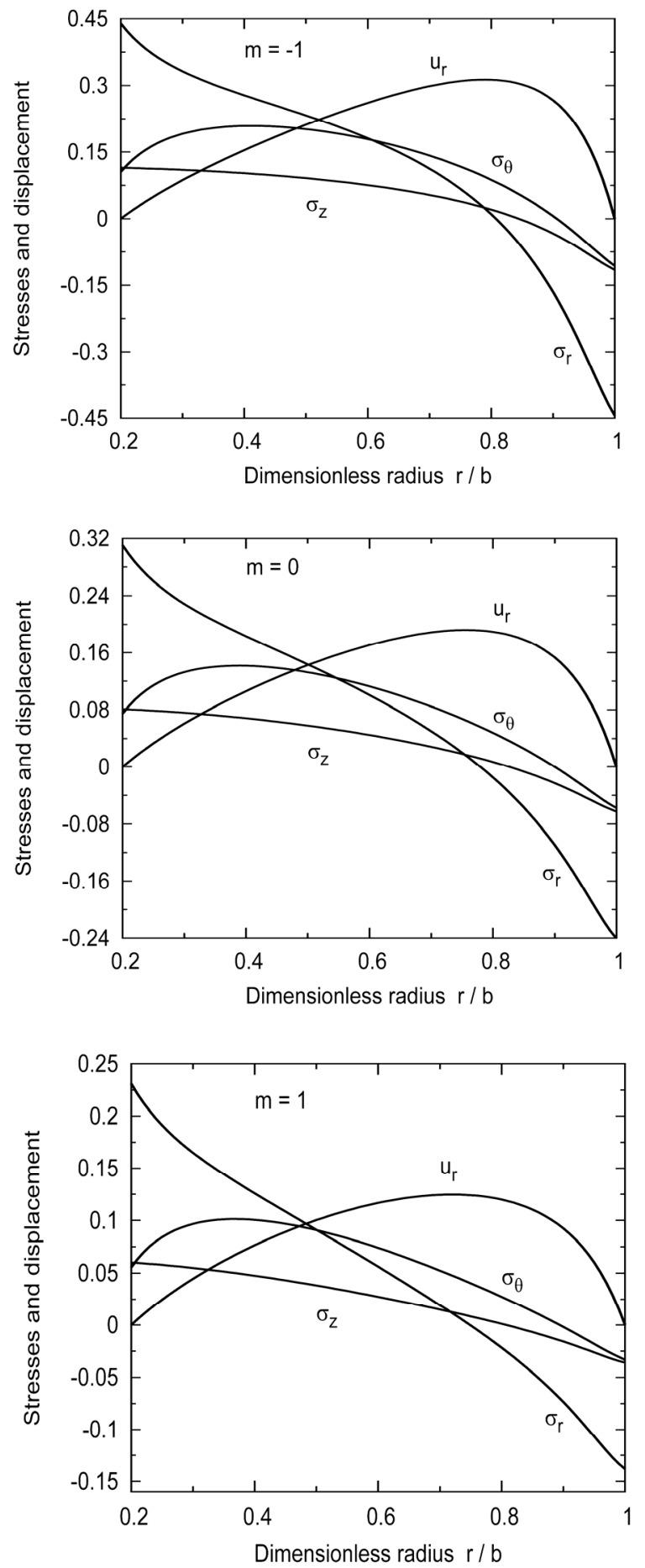

Figure 5. Distribution of dimensionless stresses and displacement through the radial direction of a CC variablethickness viscoelastic hollow cylinder. 

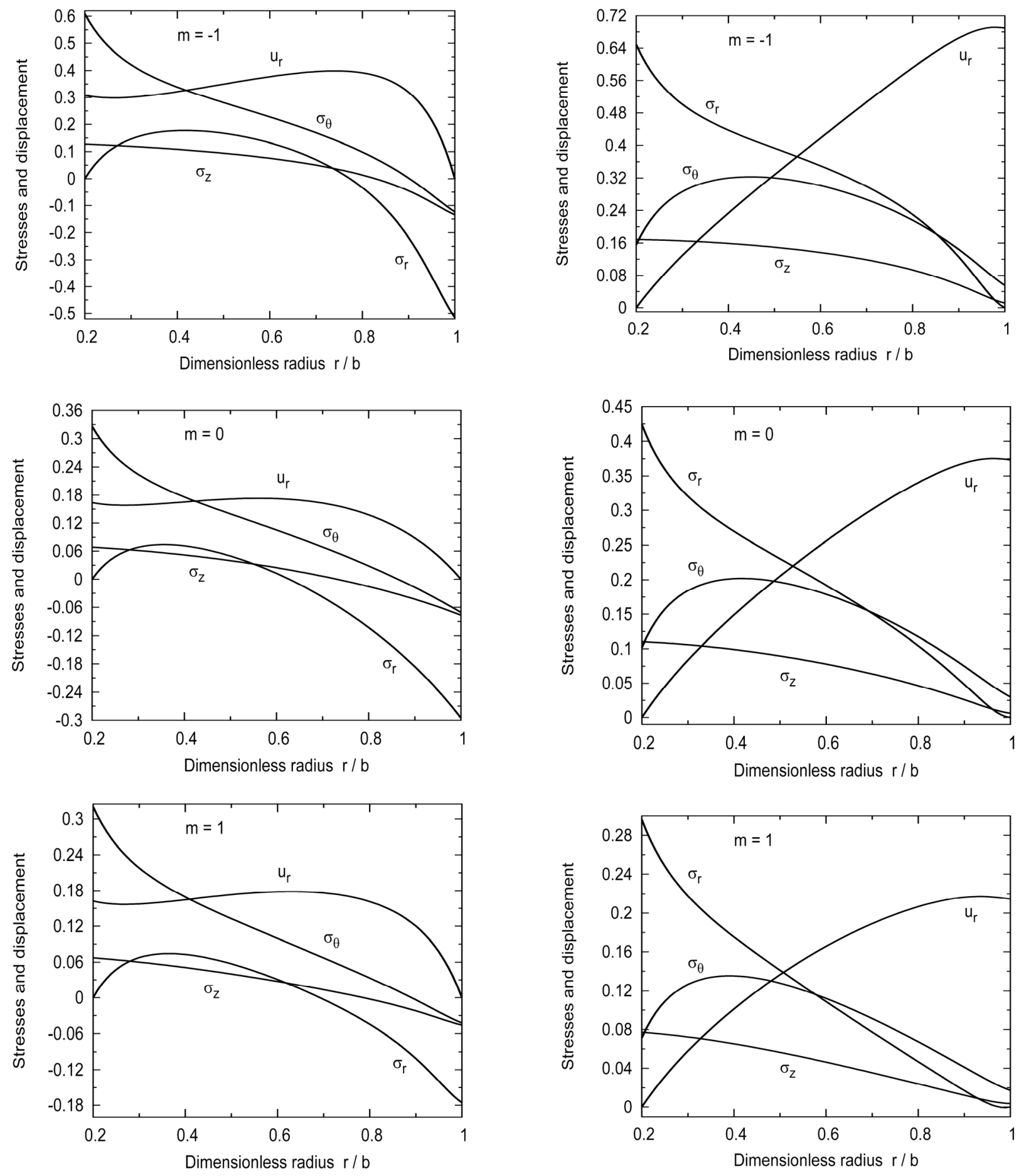

Figure 6. Distribution of dimensionless stresses and displacement through the radial direction of a FC variablethickness viscoelastic hollow cylinder.

viscoelastic inhomogeneous cylinder subjected to various boundary conditions with different values of the parameter $m$. The stresses and displacement for $m=1$ are the smallest when compared to the results for $m=0$

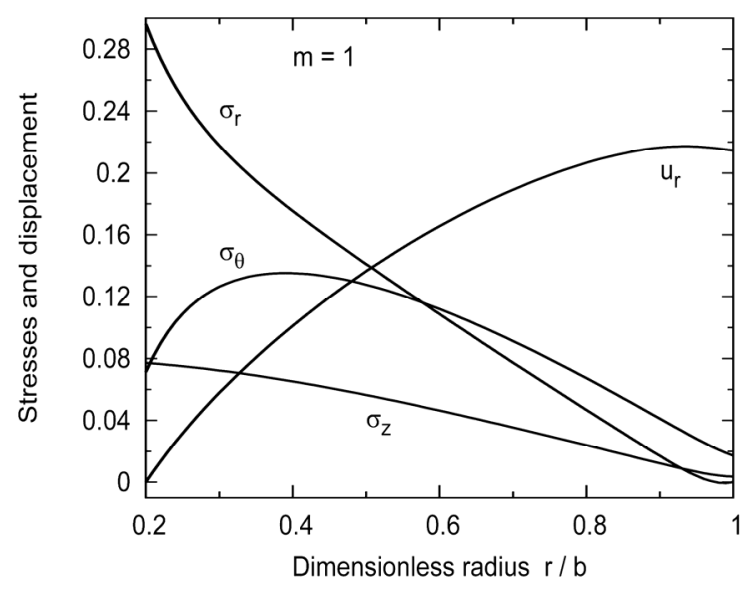

Figure 7. Distribution of dimensionless stresses and displacement through the radial direction of a CF variablethickness viscoelastic hollow cylinder.

and -1 . For FF and FC hollow cylinders, the dimensionless radial displacement $u_{r}$ has changed concavity. The dimensionless radial stress $\sigma_{r}$ increases firstly to get its maximum value then it decreases again at the 
external surface to get zero value for FF boundary condition while it tends to a constant value for FC boundary condition. In both cases, the dimensionless circumferential stress $\sigma_{\theta}$ has maximum value at the inner surface. Also, the dimensionless radial displacement $u_{r}$ increases directly as the dimensionless radius $\bar{r}$ increases for CF hollow cylinders while the highest values of it occur near the external surfaces of the CC hollow cylinders. The dimensionless radial stress $\sigma_{r}$ is monotone decreasing in $\bar{r}$ for CC and CF hollow cylinders. In all figures, the dimensionless axial stress $\sigma_{z}$ decreases from the inner to the outer surface. Also, the dimensionless radial displacement for a profile $k=0.6$, $n=0.8$ and $m=1$ is plotted in Figure 8 with various values of the constitutive parameter $p$. For CF and FF hollow cylinders, the dimensionless radial displacement $u_{r}$ and the concavity changed of it for FC hollow cylinder increase with the decreasing of the constitutive parameter $p$. In addition, the maximum values of $u_{r}$ decrease with the increase of $p$ for CC hollow cylinder. Note that, the maximum values of $u_{r}$ occur at the same
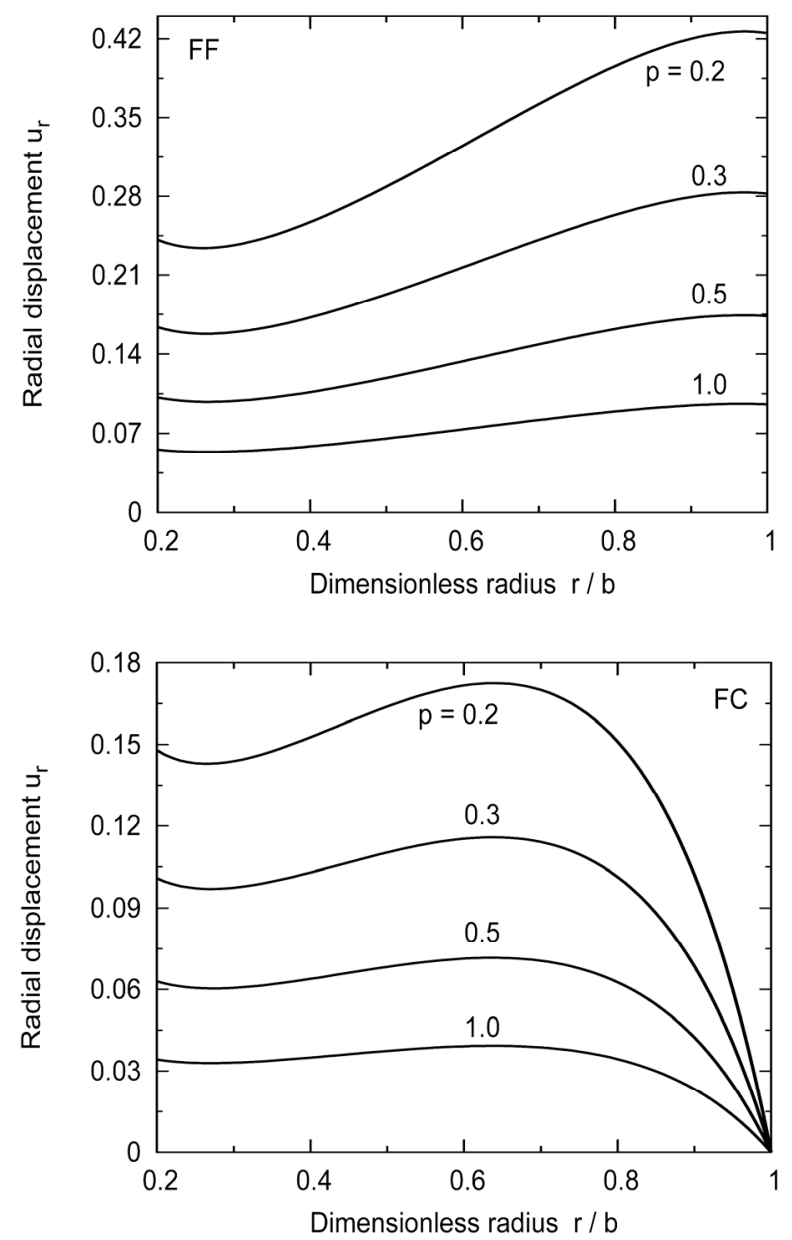

position, $\bar{r}=0.72$ for different values of $p$.

Finally, the influence of time parameter $\tau$ on the dimensionless displacement and stresses for variable thickness viscoelastic hollow cylinder subjected to FF, CC, FC and CF boundary conditions is plotted in Figure 9. This influence is studied at the position $\bar{r}=0.5$ with geometric parameters $k=0.6, n=0.8$ and $m=1$. For all hollow cylinders, the dimensionless radial displacement $u_{r}$ increases rapidly with increasing the time parameter $\tau$ to get a constant value for $\tau \geq 55$. Also for FF hollow cylinders, the dimensionless radial $\sigma_{r}$ and circumferential $\sigma_{\theta}$ stresses may be unchanged with time parameter $\tau \geq 2.5$ while the dimensionless axial stress $\sigma_{z}$ increases rapidly to still unchanged for $\tau \geq 8$. For CC and FC hollow cylinders, the highest values of $\sigma_{r}, \sigma_{\theta}$ and $\sigma_{z}$ occur at $\tau \approx 3,2.5$ and 5, respectively, then they are decreasing in the intervals $3<\tau<14$, $2.5<\tau<16$ and $5<\tau<17$ to still unchanged for $\tau \geq 14,16$ and 17, respectively. Also for CF hollow cylinder, the minimum value of the dimensionless radial stress happens at $\tau \approx 2$ then it is increasing slowly to app-
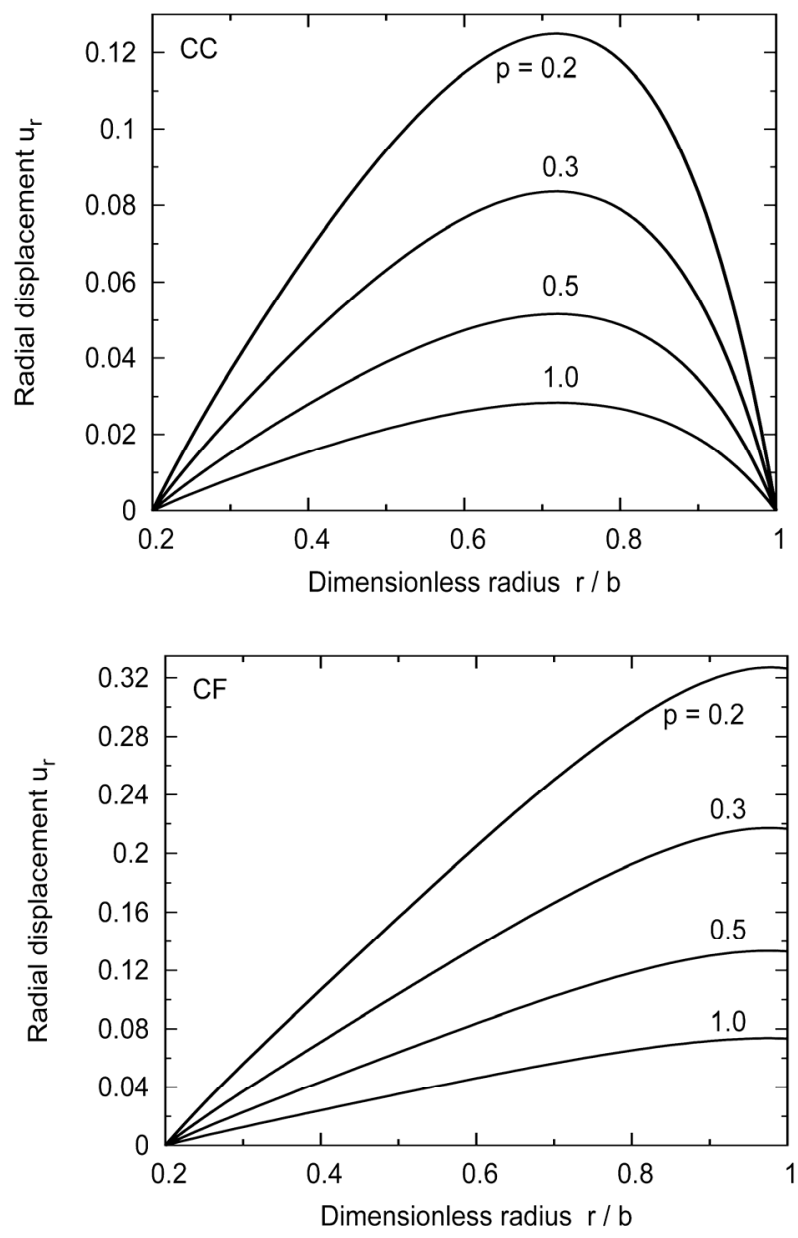

Figure 8. The effect of the constitutive parameter $p$ on $u_{r}$ of a variable-thickness viscoelastic hollow cylinder. 

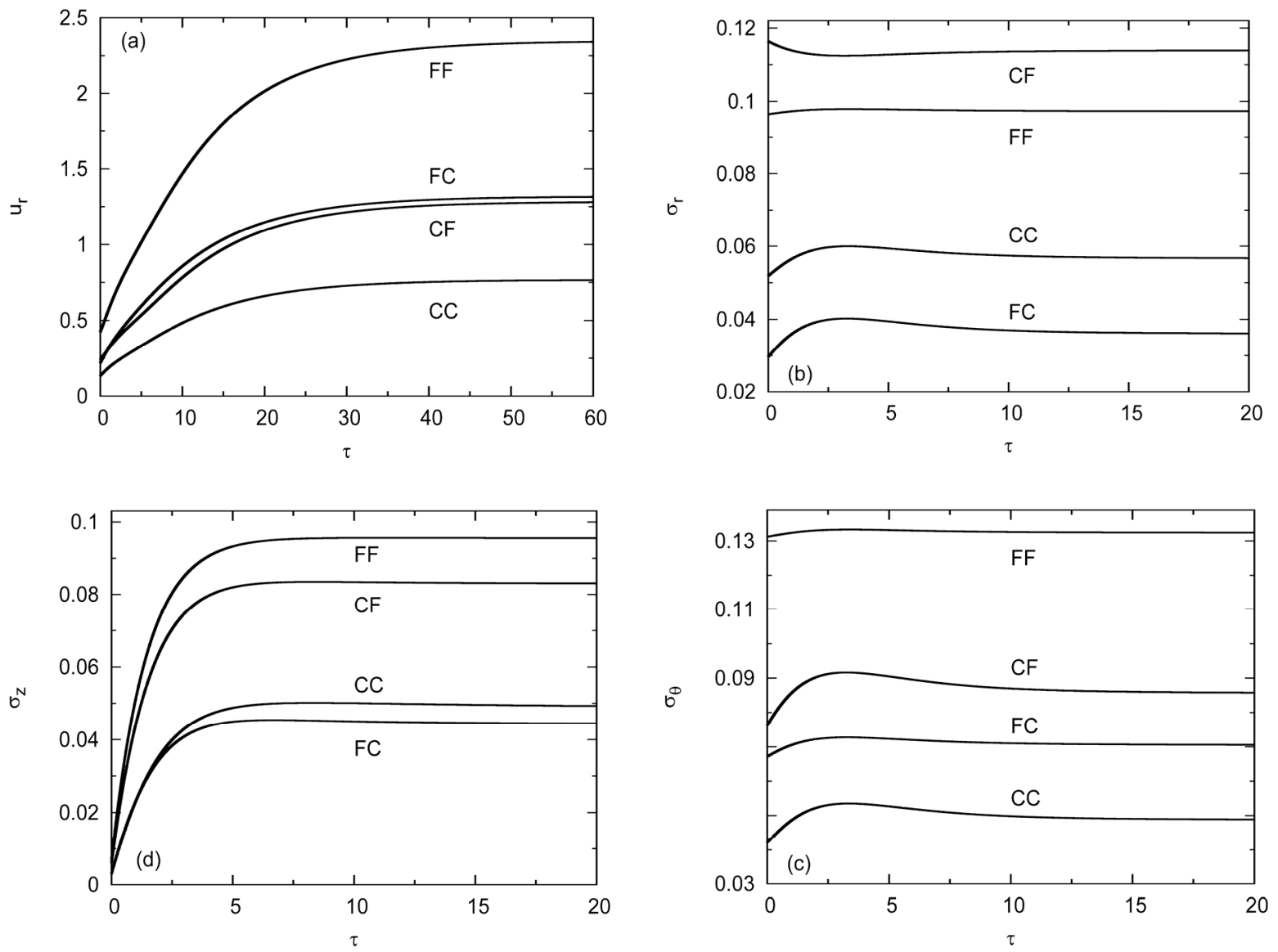

Figure 9. The effect of time parameter $\tau$ on (a) $u_{r}$, (b) $\sigma_{r}$, (c) $\sigma_{\theta}$ and (d) $\sigma_{z}$ of a variable-thickness viscoelastic hollow cylinder at $\bar{r}=\mathbf{0 . 5}$.

roach a constant value for $\tau \geq 13$. However, the dimensional circumferential and axial stresses increase to get their maximums at $\tau \approx 3.5$ and 7.5 , respectively, then decrease to still unchanged for $\tau \geq 15$ and 17.5 , respectively.

\section{Conclusions}

The rotation problem of a fiber-reinforced viscoelastic inhomogeneous variable-thickness hollow cylinder has been studied. The elastic problem is solved analytically by using the hypergeometric functions. The viscoelastic problem is solved using both the method of effective moduli and Illyushin's approximation method. Analytical solution for rotating fiber-reinforced viscoelastic inhomogeneous anisotropic hollow cylinder of variable thickness and density subjected to different boundary conditions are derived. The displacement and stresses for rotating fiber-reinforced viscoelastic homogeneous isotropic hollow cylinder with uniform thickness and density are obtained as special cases of the investigated problem. The effects due to many parameters on the radial dis- placement and stresses are investigated.

\section{References}

[1] I. E. Troyanovskii and M. A. Koltunov, "Temperature Stresses in a Long Hollow Viscoelastic Cylinder with Variable Inner Boundary,” Mekhanika Polimerov, Vol. 5, No. 2, 1969, pp. 219-226.

[2] M. A. Koltunov and I. E. Troyanovskii, "State of Stress of a Hollow Viscoelastic Cylinder Whose Material Properties Depend on Temperature," Mechanics of Composite Materials, Vol. 6, No. 1, 1970, pp. 72-79.

[3] E. C. Ting and J. L. Tuan, "Effect of Cyclic Internal Pressure on the Temperature Distribution in a Viscoelastic Cylinder," International Journal of Mechanical Sciences, Vol. 15, No. 11, 1973, pp. 861-871.

[4] L. Kh. Talybly, "Deformation of a Viscoelastic Cylinder Fastened to a Housing under Non-Isothermal Dynamic Loading," Journal of Applied Mathematics and Mechanics, Vol. 54, No. 1, 1990, pp. 74-82.

[5] W. W. Feng, T. Hung and G. Chang, "Extension and Torsion of Hyperviscoelastic Cylinders,” International Journal of Non-Linear Mechanics, Vol. 27, No. 3, 1992, pp. 329-335. 
[6] V. G. Karnaukhov and I. K. Senchenkov, "ThermomeChanical Behavior of a Viscoelastic Finite Circular Cylinder under Harmonic Deformations,” Journal of Engineering Mathematics, Vol. 46, No. 3-4, 2003, pp. 299-312.

[7] D. Bland, “The Linear Theory of Viscoelasticity,” Pergamon Press, New York, 1960.

[8] D. Abolinsh, "Elasticity Tensor for Unidirectionally Reinforced Elastic Material,” Polymer Mechanics, Vol. 4, No. 1, 1965, pp. 25-59.

[9] A. A. Illyushin and B. E. Pobedria, "Foundations of Mathematical Theory of Thermo-Viscoelasticity,” in Russian, Nauka, Moscow, 1970.

[10] M. N. M. Allam and P. G. Appleby, "On the Plane Deformation of Fiber-Reinforced Viscoelastic Plates," Applied Mathematical Modelling, Vol. 9, No. 5, 1985, pp. 341-346.

[11] M. N. M. Allam and P. G. Appleby, "On the Stress Concentrations around a Circular Hole in a Fiber-Reinforced Viscoelastic Plate,” Res Mechanica, Vol. 19, No. 2, 1986, pp. 113-126.

[12] M. N. M. Allam and A. M. Zenkour, "Bending Response of a Fiber-Reinforced Viscoelastic Arched Bridge Model,” Applied Mathematical Modelling, Vol. 27, No. 3, 2003, pp. 233-248.

[13] A. M. Zenkour and M. N. M. Allam, "Stresses around Filled and Unfilled Circular Holes in a Fiber-Reinforced Viscoelastic Plate under Bending," Mechanics of Advanced Materials and Structures, Vol. 12, No. 6, 2005, pp. 379-389.
[14] A. M. Zenkour and M. N. M. Allam, "On the Rotating Fiber-Reinforced Viscoelastic Composite Solid and Annular Disks of Variable Thickness,” International Journal for Computational Methods in Engineering Science and Mechanics, Vol. 7, No. 1, 2006, pp. 21-31.

[15] M. N. M. Allam, A. M. Zenkour and H. F. El-Mekawy, "Stress Concentrations in a Viscoelastic Composite Plate Weakened by a Triangular Hole,” Composite Structures, Vol. 79, No. 1, 2007, pp. 1-11.

[16] A. M. Zenkour, K. A. Elsibai and D. S. Mashat, "Elastic and Viscoelastic Solutions to Rotating Functionally Graded Hollow and Solid Cylinders,” Applied Mathematics and Mechanics - English Edition, Vol. 29, No. 12, 2008, pp. 1601-1616.

[17] A. E. Bogdanovich and C. M Pastore, "Mechanics of Textile and Laminated Composites with Applications to Structural Analysis,” Chapman and Hall, New York, 1996.

[18] B. E. Pobedria, "Structural Anisotropy in Viscoelasticity," Mechanics of Composite Materials, Vol. 12, No. 4, 1976, pp. 557-561.

[19] A. M. Zenkour, "Thermal Effects on the Bending Response of Fiber-Reinforced Viscoelastic Composite Plates using a Sinusoidal Shear Deformation Theory," Acta Mechanica, Vol. 171, No. 3-4, 2004, pp. 171-187.

[20] M. N. M. Allam and B. E. Pobedria, "On the Solution of Quasi-Statical Problems of Anisotropic Viscoelasticity," Izv. Acad. Nauk ArSSR, In Russian, Mechanics, Vol. 31, No. 1, 1978, pp. 19-27. 